\title{
Multicomponent Crystal of Trimethoprim and Citric Acid: Solid State Characterization and Dissolution Rate Studies
}

\author{
Salman Umar, Rido Farnandi, Hulwa Salsabila, Erizal Zaini* \\ Department of Pharmaceutics, Faculty of Pharmacy, Universitas Andalas, Padang, Indonesia
}

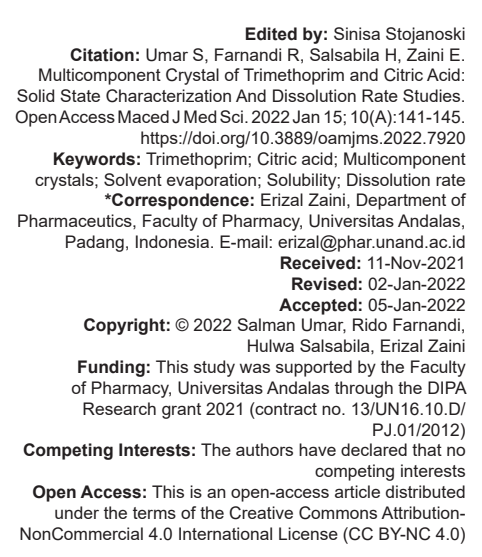

Introduction

Solubility is one of the most important physicochemical properties of drug compounds in predicting drug absorption in the gastrointestinal tract. Drugs that have low solubility in water often show low bioavailability and dissolution rate is a determining step in the drug absorption process [1]. One of the active pharmaceutical ingredients with low solubility properties is trimethoprim. According to Biopharmaceutical Classification System, trimethoprim is classified under class II along with other drugs with low solubility and high permeability [2]. Trimethoprim acts by inhibiting enzyme dihydrofolate reductase in the reduction of dihydrofolate to tetrahydrofolate which causes bacteria to lack essential di- and tetrahydrofolic acids in their biosynthesis [3]. Trimethoprim is administered through various routes, including intravenous, intramuscular, and oral. However, due to its low solubility, oral solid preparation of trimethoprim has low bioavailability even though it has high gastrointestinal tolerability and low side effects [4]. Previous studies have reported several approaches in attempt to enhance solubility and dissolution rate of trimethoprim, including solid dispersion system with hydrophilic polymers, inclusion complexes formation with cyclodextrins, and spherical crystallization [2], [4], [5].

One of the recent strategies applied to enhance physicochemical properties of active pharmaceutical compounds is by forming a multi-component crystal phase with an inert and safe coformer. This approach has shown successful improvement in solubility, dissolution rate, physical and chemical stability, and compressibility [6], [7], [8], [9], [10]. Multicomponent crystal phase between active pharmaceutical compounds and coformers could be formed due to non-covalent intermolecular interactions such as van der Waals bonds and hydrogen bonds [11].

Several multi-component crystal phases of trimethoprim include cocrystals, some of which have been reported including salts with sulfamethoxazole, mefenamic acid, cinnamic acid, formic acid, acetic acid, malic acid, and maleic acid [8], [12], [13], [14], [15], [16], [17]. However, so far there has been no report of multicomponent crystal phases of trimethoprim with citric acid. Citric acid is a safe excipient categorized by the food and drug administration as generally recognized as safe. This study aimed to prepare a multicomponent crystal phase of trimethoprim with citric acid by the solvent 
evaporation method. Solid-state properties were characterized by powder X-ray diffraction (PXRD), differential scanning calorimetry (DSC), scanning electron microscopy (SEM), and FT-IR spectroscopy. Solubility and dissolution rate were evaluated in aqueous medium, compared to intact trimethoprim.

\section{Material and Methods}

\section{Materials}

Materials used include trimethoprim (Wako Pure Chemical Industries, Japan), citric acid (TCl-EP, Tokyo Japan), ethanol (Merck, Germany), methanol (Merck, Germany), hydrochloric Acid (Bratachem, Indonesia), and $\mathrm{CO}_{2}$-free distilled water (Brataco).

\section{phase}

\section{Preparation of multicomponent crystal}

Multi-component crystals of trimethoprim and citric acid were prepared by solvent evaporation method with an equimolar ratio $(0.290 \mathrm{~g}: 0.192 \mathrm{~g})$. Then trimethoprim was dissolved with methanol and citric acid was dissolved in ethanol, then the two materials were quickly mixed at $85 \mathrm{rpm}$ in a magnetic stirrer. After that, it was dried in a desiccator to form a crystalline solid.

\section{PXRD analysis}

Analyses were carried out on trimethoprim, citric acid, and multi-component crystals. X-ray diffraction analyses of the samples were performed at room temperature using an X-ray diffractometer (Philips X'Pert Powder, The Netherland) with CuKradiation $(\lambda=1.54178 \AA)$, current $40 \mathrm{~mA}$, voltage $40 \mathrm{kV}$. Samples were measured in reflection mode at 0.05 theta with an angle range of $5^{\circ}-40^{\circ}$ theta at a scan speed of $5 \% \mathrm{~min}$.

\section{Analysis of DSC}

Thermal analyses on trimethoprim, citric acid, and multicomponent crystal compounds were carried out using a temperature-calibrated DSC tool. Samples were placed in a closed aluminum pan. The DSC device (DSC-60 Plus Shimadzu, Japan), is programmed in a temperature range of $30-250^{\circ} \mathrm{C}$, heating speed of $10^{\circ} \mathrm{C} / \mathrm{min}$, under nitrogen gas flow of about $30 \mathrm{Psi}$.

\section{FT-IR spectroscopic analysis}

Analysis was carried out on trimethoprim, citric acid, and multi-component crystals. A small amount of sample (2-3 mg) was mixed with $\mathrm{KBr}$ after which it was placed in the sample holder of the FT-IR spectroscopic instrument (IRT Racer-100 Shimadzu, Japan) and the samples were analyzed at room temperature. The spectrum was measured in the range of $4500-500 \mathrm{~cm}^{-1}$ wavenumber.

\section{SEM analysis}

SEM (Hitachi Type S-3400N, Japan) analysis was performed on trimethoprim, citric acid, and multicomponent crystals. The samples were coated with a thin layer of palladium-gold prior to analysis. SEM works using a beam speed of $30 \mathrm{kV}$.

\section{Solubility test}

In the solubility test, an excess amount of trimethoprim and multicomponent crystals were added to $100 \mathrm{ml}$ of CO2-free distilled water, stirred at a speed of $150 \mathrm{rpm}$ for 24 hours at room temperature, and then filtered using filter paper. Concentration of trimethoprim was determined from the absorbance measurement at $287 \mathrm{~nm}$ using ultraviolet-visible light (UV-Vis) spectrophotometer.

\section{Dissolution rate profile study}

The dissolution rate study of trimethoprim and multicomponent crystals used the paddle method (Hanson Research SR08, USA) at $37 \pm 0.5^{\circ} \mathrm{C}$ at a speed of $100 \mathrm{rpm}$ for $60 \mathrm{~min}$ with two mediums namely $0.1 \mathrm{~N} \mathrm{HCl}$ and $\mathrm{CO}_{2}$-free distilled water. Five $\mathrm{mL}$ of each dissolution medium was pipetted at $5,10,15,30,45$, $60 \mathrm{~min}$. The absorbance of the solution that had been pipetted from the dissolution medium was measured using UV-visible spectrophotometer (at $287 \mathrm{~nm}$ ) to determine the amount of trimethoprim dissolved.

\section{Results and Discussion}

X-ray diffraction analysis is a technique to characterize the solid properties of active pharmaceutical compounds. Changes in the X-ray diffraction pattern in solids resulting from intramolecular interactions indicate the formation of new crystalline phases such as cocrystals or salts [18]. Figure 1 presents the X-ray diffraction pattern of trimethoprim, citric acid, and the multi-component crystalline phase. Figure $1 \mathrm{a}$ is an X-ray diffraction pattern of trimethoprim showing typical diffraction peaks at 2 theta $=11.62 ; 12.86 ; 16.12 ; 25.46$ and 28.45 . Figure $1 \mathrm{~b}$, is an X-ray diffraction pattern of citric acid which has a specific diffraction peak at 2 theta $=14.10 ; 17.89$; and 20.08. Figure $1 \mathrm{c}$ shows the X-ray diffraction pattern of the multi-component crystalline 


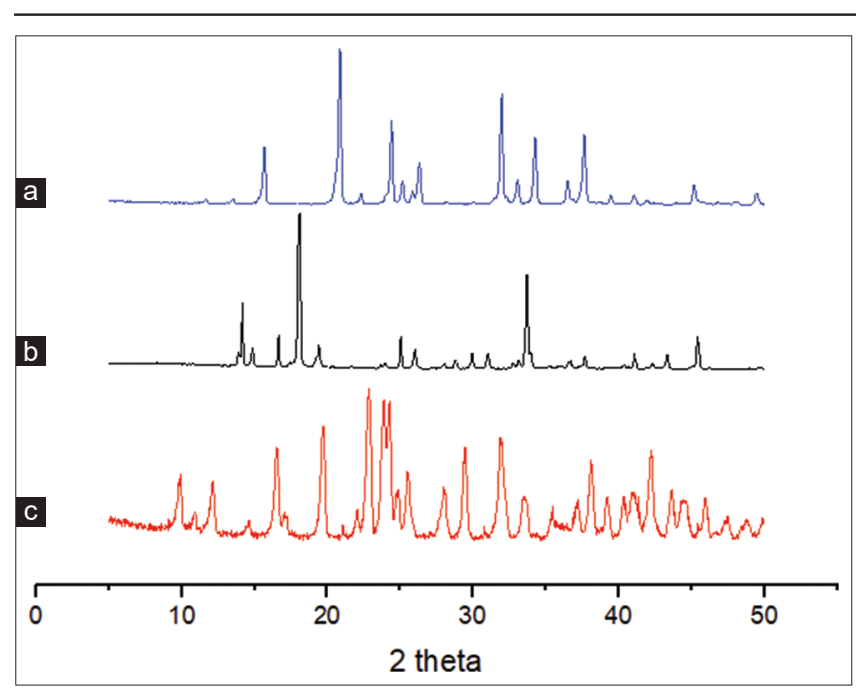

Figure 1: Powder X-ray diffraction diffractogram of (a) Trimethoprim, (b) Citric acid, and (c) Multicomponent crystals

phase which is unique and different from the X-ray diffraction pattern of trimethoprim and citric acid coformer compounds. There are several new peaks, namely at 2 theta $=13.13 ; 21.53$ and 24.01 . These results show the formation of a new crystalline phase as a consequence of the interaction between trimethoprim and citric acid. Multicomponent crystal phase between the two solid phases is very possible because of the presence of functional groups between the two molecules that can be bonded through weak non-covalent bonds. Based on the $\Delta \mathrm{pKa}$ rule theory, if the pKa difference between the active pharmaceutical compound and the coformer is greater than $(>) 3$, then the interaction can form a salt type of multicomponent crystal [19], [20]. pKa difference between trimethoprim and citric acid of 4.17 makes it possible to form a salt-type multicomponent crystal.

Thermal analysis is an analysis used to evaluate the physical and chemical properties of a substance as a function of temperature. The existence of solid-state interactions is indicated by a change in the melting point between a binary mixture of active pharmaceutical ingredients and coformers.

Figure $2 \mathrm{a}$ displays a thermogram of trimethoprim which shows an endothermic peak at

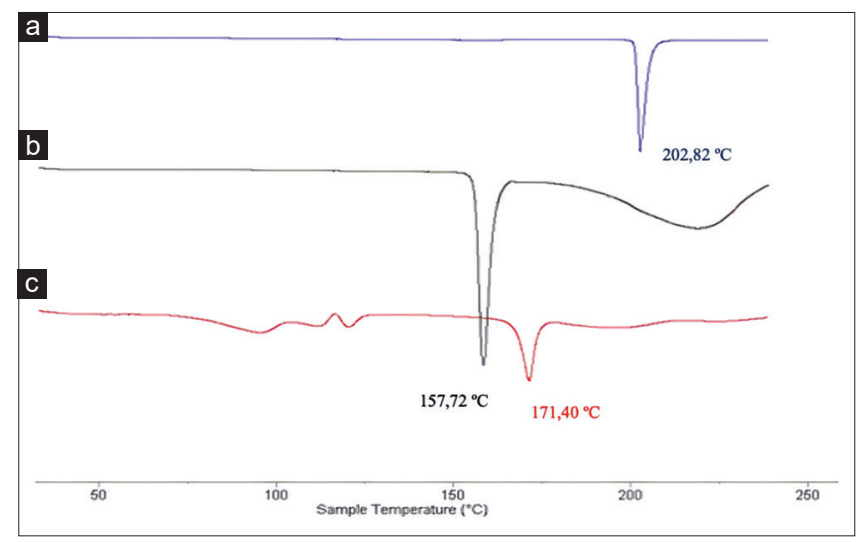

Figure 2: Differential scanning calorimetry thermogram of (a) Trimethoprim, (b) Citric acid, and (c) Multicomponent crystals $202.82^{\circ} \mathrm{C}$ as the melting point of this solid compound. Citric acid thermogram (Figure 2b) shows a single sharp endothermic peak at $157.72^{\circ} \mathrm{C}$, which is also a melting event of citric acid. Multicomponent crystals of trimethoprim-citric acid show several endothermic peaks of $90-120^{\circ} \mathrm{C}$ which is either loss of solvent or dehydration of the solid. While the sharp endothermic peak at a temperature of $171.40^{\circ} \mathrm{C}$ is the new melting point of the multi-component crystal phase. The results of the DSC thermal analysis supported the PXRD analysis, that between trimethoprim and citric acid, a new multi-component crystal phase was formed [21].

FT-IR spectroscopic analysis is one of the important techniques used to evaluate intramolecular interactions in multicomponent crystal systems. The presence of solid-state interactions between components in a crystalline multicomponent system is indicated by the presence of a new or a shifting transmittance peak. The presence of hydrogen bonds formed between the two solid substances is indicated by a shift in the wavenumber [21], [22].

Figure3displaytheFT-IRspectra of trimethoprim, citric acid, and a multi-component crystal phase. The peak transmittance of trimethoprim at wave numbers (OH) $311.19 \mathrm{~cm}^{-1}$, (NH) $3468.07 \mathrm{~cm}^{-1}$, (CH) $2929.92 \mathrm{~cm}^{-1}$, $(\mathrm{C}=\mathrm{N}) 1634.70 \mathrm{~cm}^{-1}$ and $(\mathrm{C}=\mathrm{H}) 1464,00 \mathrm{~cm}^{-1}$. Citric acid at wave number $(\mathrm{OH}) 3493.15 \mathrm{~cm}^{-1},(\mathrm{C}=\mathrm{O}) 17421.71 \mathrm{~cm}^{-1}$, $(\mathrm{C}=\mathrm{H}) 1424.45 \mathrm{~cm}^{-1}$ and the peak multicomponent crystal transmittance at wavenumber $(\mathrm{OH}) 3388.89 \mathrm{~cm}^{-1},(\mathrm{NH})$ $3573.19 \mathrm{~cm}^{-1},(\mathrm{CH} 3) 3132.45 \mathrm{~cm}^{-1},(\mathrm{CH}) 2843.12 \mathrm{~cm}^{-1}$, $(\mathrm{C}=\mathrm{O}) \quad 1734.04 \mathrm{~cm}^{-1}, \quad(\mathrm{C}=\mathrm{N}) \quad 1589.37 \mathrm{~cm}^{-1}$, and $(\mathrm{C}=\mathrm{H}) 1322.23 \mathrm{~cm}^{-1}$. The shift in the wavenumber of trimethoprim in the multicomponent crystal in the $\mathrm{OH}$ group from $3101.59 \mathrm{~cm}^{-1}$ to $3388.89 \mathrm{~cm}^{-1}$, the $\mathrm{NH}$ group from $3468.07 \mathrm{~cm}^{-1}$ to $3573.19 \mathrm{~cm}^{-1}$ the $\mathrm{CH}$ group from $2929.92 \mathrm{~cm}^{-1}$ to $2843.12 \mathrm{~cm}^{-1}$, the $\mathrm{C}=\mathrm{N}$ group from $1636.70 \mathrm{~cm}^{-1}$ to $1589.37 \mathrm{~cm}^{-1}$ and the $\mathrm{C}=\mathrm{H}$ group from 1464.00 to $1452.42 \mathrm{~cm}^{-1}$.

The results of the analysis of trimethoprim, citric acid, and multi-component crystals by SEM can

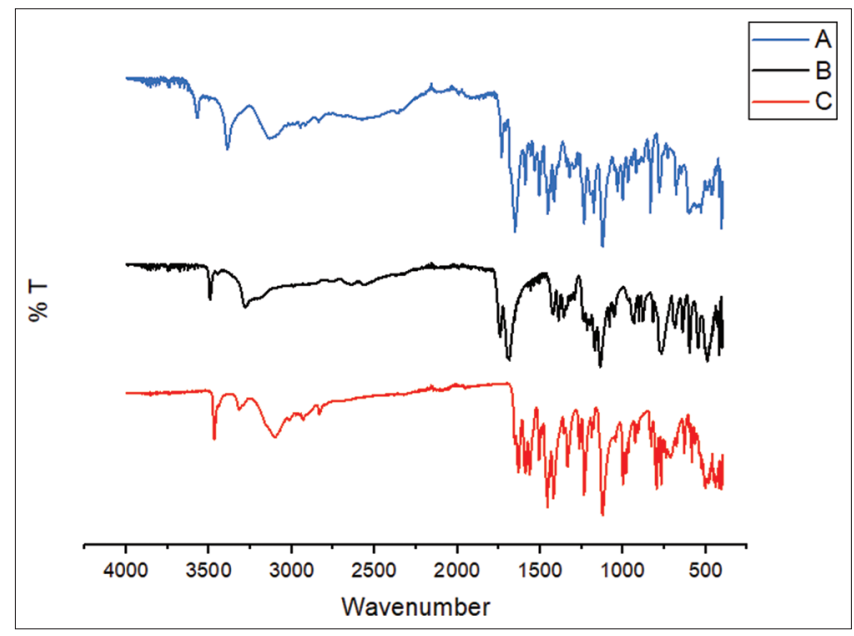

Figure 3: FT-IR Spectra of (a) trimethoprim, (b) citric acid, and (c) multicomponent crystals 
be seen in Figure 4. Based on observations, it can be seen that trimethoprim crystals are irregular cubic crystals, citric acid is rigid prismatic crystals, while multicomponent crystals show a new crystal habit as long rod-shaped.

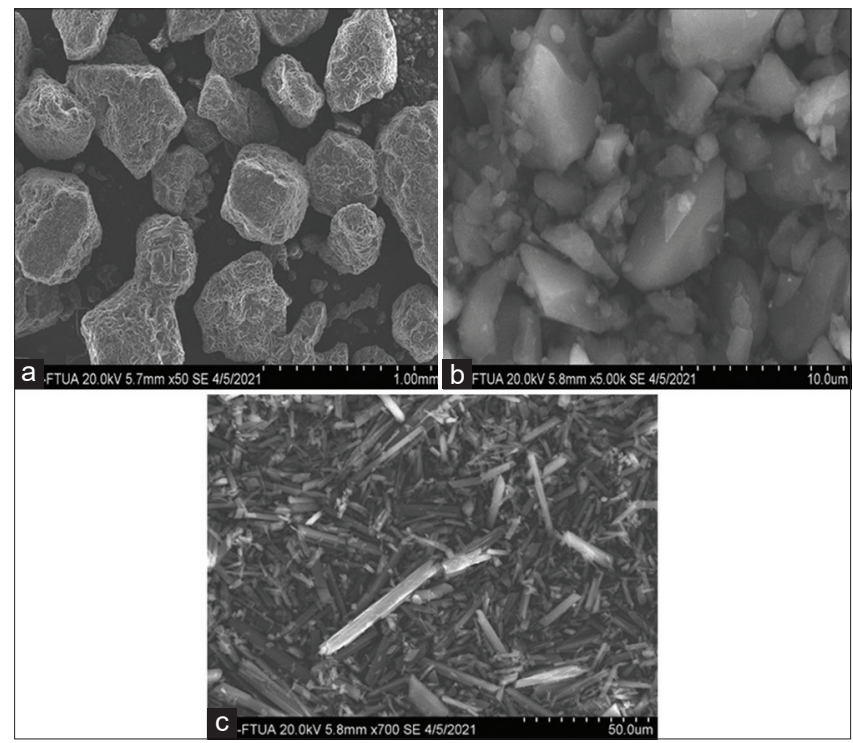

Figure 4: Scanning electron microscopy of (a) trimethoprim, (b) citric acid, and (c) multicomponent crystal

Orally administered drugs that are poorly soluble in water generally have limited bioavailability since solubility plays an important role in gastrointestinal absorption. The formation of multicomponent crystals with coformers could modify active pharmaceutical ingredients' properties and increase solubility and pharmacological effectiveness [1], [11]. The main advantage of this approach is the ability to maintain its thermodynamic stability and retain the drug in the solid crystalline phase. Moreover, this method does not change the pharmacophore structure of the active pharmaceutical ingredients [23].

Table 1: Solubility of trimethoprim and its multicomponent with citric acid $(n=3)$

\begin{tabular}{ll}
\hline Compound & Solubility \pm SD $(\mathrm{mg} / 100 \mathrm{~mL})$ \\
\hline Trimethoprim & $1.08 \pm 0.233$ \\
Multicomponent Crystals & $7.05 \pm 0.110$ \\
\hline
\end{tabular}

Solubility studies (Table 1) show that the multicomponent crystals of trimethoprim are 7 times more soluble than intact trimethoprim alone. The dissolution rate profiles of trimethoprim and multicomponent crystals were performed by paddle method at $100 \mathrm{rpm}$ for $60 \mathrm{~min}$ at $37 \pm 0.5^{\circ} \mathrm{C}$ with $0.1 \mathrm{~N}$ $\mathrm{HCl}$ medium and $\mathrm{CO}_{2}$-free distilled water. The increase in the rate profile of trimethoprim and multicomponent crystals of $0.1 \mathrm{~N} \mathrm{HCl}$ medium can be seen in Figure 5. with an increase in dissolution efficiency of 1.46 times. Moreover, the increase in the rate profile of trimethoprim and multicomponent crystalline $\mathrm{CO}_{2}$-free distilled water media can be seen in Figure 6 with an increase in dissolution efficiency of 2.45 times. The formation of salt has been proven to improve the physicochemical properties of drug, especially dissolution rate and solubility. Earlier studies have reported improved

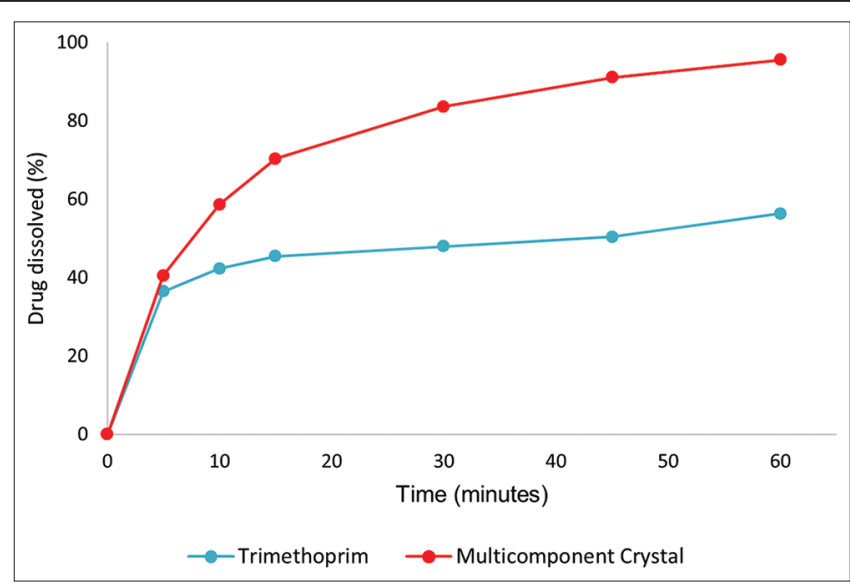

Figure 5: Dissolution rate profile of trimethoprim and multicomponent crystals in $0.1 \mathrm{~N} \mathrm{HCl}$ medium

dissolution rate and solubility of trimethoprim through multicomponent crystalline phase with several excipients [12], [13], [14], [15], [16], [17], [24].

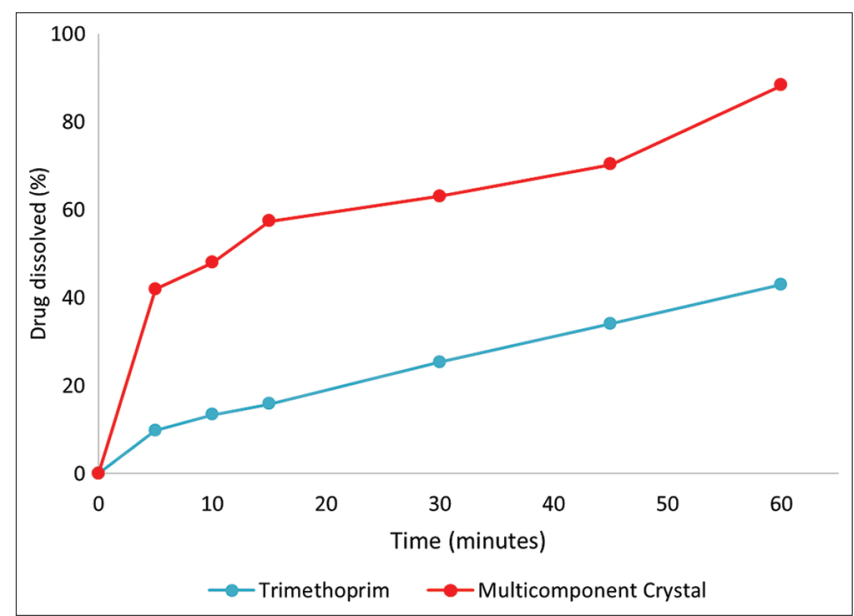

Figure 6: Dissolution rate profile of trimethoprim and multicomponent crystal in $\mathrm{CO}_{2}$-free distilled water medium

Some factors may contribute to the increment of solubility and dissolution rate of multicomponent crystals of trimethoprim and citric acid. First, solid phase of multicomponent crystals is more hydrophilic so it has a better affinity for the aqueous medium. The salt form dissociates into cationic and anionic ions once in contact with aqueous media. Second, in terms of solid-state properties, lower melting point of the crystal phase and change in the crystal structure indicates weaker lattice energy that binds the molecules in the unit cell. Weaker lattice energy of the crystal phase leads to a faster dissolution rate [8], [19], [23].

\section{Conclusions}

In this current research, the salt-type multicomponent crystal of trimethoprim and citric acid were successfully prepared and characterized its solid-state properties. Novel trimethoprim - citric acid 
multicomponent crystal phase significantly improves solubility and dissolution rate of trimethoprim in compared to intact trimethoprim.

\section{References}

1. Kawabata $\mathrm{Y}$, Wada K, Nakatani M, Yamada S, Onoue S. Formulation design for poorly water-soluble drugs based on biopharmaceutics classification system: Basic approaches and practical applications. Int J Pharm. 2011;420(1):1-10. https://doi. org/10.1016/j.ijpharm.2011.08.032

PMid:21884771

2. Li N, Zhang YH, Wu YN, Xiong XL, Zhang YH. Inclusion complex of trimethoprim with beta-cyclodextrin. J Pharm Biomed Anal. 2005;39(3-4):824-9. https://doi.org/10.1016/j.jpba.2005.05.011 PMid: 16011886

3. Hawser S, Lociuro S, Islam K. Dihydrofolate reductase inhibitors as antibacterial agents. Biochem Pharmacol. 2006;71(7):941-8. https://doi.org/10.1016/j.bcp.2005.10.052

PMid:16359642

4. Guptat RL, Kumar R, Singla AK. Enhanced dissolution and absorption of trimethoprim from coprecipitates with polyethylene glycols and polyvinylpyrrolidone. Drug Dev Ind Pharm. 1991;17(3):463-8.

5. Pawar PH, Pawar AP, Mahadik KR, Paradkar AR. Evaluation of tableting properties of agglomerates obtained by spherical crystallisation of trimethroprim. Indian J Pharm Sci. 1998;60(1):24-8.

6. Nugrahani I, Fisandra F, Horikawa A, Uekusa H. New sodium mefenamate-nicotinamide multicomponent crystal development to modulate solubility and dissolution: Preparation, structural, and performance study. J Pharm Sci. 2021;110(9):324-60. https://doi.org/10.1016/j.xphs.2021.05.022 PMid:34090898

7. Zaini E, Afriyani A, Fitriani L, Ismed F, Horikawa A, Uekusa H. Improved solubility and dissolution rates in novel multicomponent crystals of piperine with succinic acid. Sci Pharm. 2020;88(2):21.

8. Yuliandra $Y$, Izadihari R, Rosaini H, Zaini E. Multicomponent crystals of mefenamic acid-tromethamine with improved dissolution rate. J Res Pharm. 2019;23(6):988-96.

9. Thakur TS, Thakuria R. Crystalline multicomponent solids: An alternative for addressing the hygroscopicity issue in pharmaceutical materials. Cryst Growth Des. 2020;20(9):6245-65.

10. Ainurofiq A, Mauludin R, Mudhakir D, Umeda D, Soewandhi SN, Putra OD, et al. Improving mechanical properties of desloratadine via multicomponent crystal formation. Eur J Pharm Sci. 2018;111:65-72. https://doi.org/10.1016/j.ejps.2017.09.035 PMid:28958892

11. Putra OD, Uekusa H. Pharmaceutical multicomponent crystals: Structure, design, and properties. In: Advances in Organic Crystal Chemistry. Singapore: Springer; 2020. p. 153-84.

12. Zaini E. Formation and characterization of sulfamethoxazoletrimethoprim cocrystal by milling process. J Appl
Pharm Sci. 2018;7(12):169-73. https://doi.org/10.7324/ JAPS.2017.71224

13. Muthiah PT, Francis S, Rychlewska U, Warzajtis B. Crysta engineering of analogous and homologous organic compounds: Hydrogen bonding patterns in trimethoprim hydrogen phthalate and trimethoprim hydrogen adipate. Beilstein J Org Chem. 2006;2:8. https://doi.org/10.1186/1860-5397-2-8 PMid:16603061

14. Bryan RF, Haltiwanger RC, Woode MK. Trimethoprim acetate. Acta Crystallogr Sect C. 1987;43(12):2412-5.

15. Umadevi B, Prabakaran P, Muthiah PT. A pseudo-quadruple hydrogen-bonding motif consisting of six N-H.O hydrogen bonds in trimethoprim formate. Acta Crystallogr C. 2002;58(8):0510-2. https://doi.org/10.1107/s0108270102011150

PMid:12154314

16. Prabakaran P, Robert J, Muthiah P, Bocelli G, Righi L. Aminopyrimidine-carboxyl(ate) interactions in trimethoprim maleate, an antifolate drug. Acta Crystallogr C. 2001;57(4):45961. https://doi.org/10.1107/s0108270101000269 PMid:11313594

17. Bhattacharya B, Das S, Lal G, Soni S, Ghosh A, Reddy C et al. Screening, crystal structures and solubility studies of a series of multidrug salt hydrates and cocrystals of fenamic acids with trimethoprim and sulfamethazine. J Mol Struct. 2019;1199:127028.

18. Al Rahal O, Williams PA, Hughes CE, Kariuki BM, Harris KD. Structure determination of multicomponent crystalline phases of (S)-ibuprofen and I-proline from powder X-ray diffraction data, augmented by complementary experimental and computational techniques. Cryst Growth Des. 2021;21(4):2498-507.

19. Zaini E, Fitriani L, Sari RY, Rosaini H, Horikawa A, Uekusa H. Multicomponent crystal of mefenamic acid and n-methyl-dglucamine: Crystal structures and dissolution study. J Pharm Sci. 2019;108(7):2341-8. https://doi.org/10.1016/j.xphs.2019.02.003 PMid:30779887

20. Ma D, Pei T, Bai Y, Zhou L, Bao Y, Yin Q, et al. Salts formation between ibuprofen and pyridine derivatives: Effect of amino group on supramolecular packing and proton transfer. $\mathrm{J} \mathrm{Mol}$ Struct. 2019;1179:487-94.

21. Martins F, Guimarães F, Honorato S, Ayala A, Ellena J. Vibrational and thermal analyses of multicomponent crystal forms of the anti-HIV drugs lamivudine and zalcitabine. J Pharm Biomed Anal. 2015;110:76-82. https://doi.org/10.1016/j. jpba.2015.03.004 PMid:25808817

22. Ishihara S, Hattori $Y$, Otsuka M, Sasaki T. Cocrystal formation through solid-state reaction between ibuprofen and nicotinamide revealed using $\mathrm{THz}$ and IR spectroscopy with multivariate analysis. Crystals. 2020;10(9):760.

23. Dwichandra Putra O, Umeda D, Fujita E, Haraguchi T, Uchida T, Yonemochi E, et al. Solubility improvement of benexate through salt formation using artificial sweetener. Pharmaceutics. 2018;10(2):64. https://doi.org/10.3390/pharmaceutics10020064 PMid:29861459

24. Yuliandra Y, Hutabarat LJ, Ardila R, Octavia MD, Zaini E. Enhancing solubility and antibacterial activity using multicomponent crystals of trimethoprim and malic acid. Pharm Educ. 2021;21(2):296-304. 